\title{
The possibility of an intrinsic spin lattice in high-mobility semiconductor heterostructures
}

\author{
CHRISTOPH SIEGERT ${ }^{1 *}$, ARINDAM GHOSH ${ }^{1,2 *}{ }^{2}$, MICHAEL PEPPER ${ }^{1}$, IAN FARRER ${ }^{1}$ AND DAVID A. RITCHIE ${ }^{1}$ \\ ${ }^{1}$ Cavendish Laboratory, University of Cambridge, J.J. Thomson Avenue, Cambridge CB3 OHE, UK \\ ${ }^{2}$ Department of Physics, Indian Institute of Science, Bangalore 560 012, India \\ *e-mail: cs423@cam.ac.uk; arindam@physics.iisc.ernet.in
}

Published online: 11 March 2007; doi:10.1038/nphys559

Embedding magnetic moments into semiconductor heterostructures offers a tuneable access to various forms of magnetic ordering and phase transitions in low-dimensional electron systems. In general, the moments are introduced artificially, by either doping with ferromagnetic atoms, or electrostatically confining odd-electron quantum dots ${ }^{1-4}$. Here, we report experimental evidence of an independent, and intrinsic, source of localized spins in high-mobility GaAs/AlGaAs heterostructures with large setback distance $(\approx 80 \mathrm{~nm})$ in modulation doping. Measurements reveal a quasi-regular distribution of the spins in the delocalized Fermi sea, and a mutual interaction via the Ruderman-Kittel-Kasuya-Yosida (RKKY) indirect exchange below $100 \mathrm{mK}$. We show that a simple model on the basis of the fluctuations in background potential on the host two-dimensional electron system can explain the observed results quantitatively, which suggests a 'disordertemplated' microscopic origin of the localized moments.

Local fluctuations in the conduction band often lead to localization of single-electron states in a dilute metallic system. These 'impurity' states are physically separated from the surrounding delocalized Fermi sea by a tunnel barrier, and can become magnetic for large on-site Coulomb interaction $U>-\epsilon$, where $\epsilon(<0)$ is the energy of the localized states relative to the surrounding Fermi level. In the limit of small valency fluctuations, that is, $-\epsilon / \Gamma \gg 1$, where $\Gamma$ is the level broadening due to finite tunnelling, the Kondo representation of the Anderson impurity model $^{5}$ allows the impurity spin to form a spin singlet with the local spin cloud of conduction electrons as the system is cooled below the characteristic Kondo temperature $T_{\mathrm{K}} \approx E_{\mathrm{F}} \exp [\pi \epsilon(\epsilon+U) / 2 \Gamma U]$, where $E_{\mathrm{F}}$ is the Fermi energy of the conduction electrons.

Because the Kondo effect contributes one additional state at each impurity site within a small bandwidth of $T_{\mathrm{K}}$ around $E_{\mathrm{F}}$, spectroscopic techniques based on tunnelling ${ }^{6}$ or non-equilibrium transport ${ }^{1-4,7-9}$ reveal a resonance in the low-energy density of states (DOS) at zero source-drain bias $\left(V_{\mathrm{SD}}\right)$. This is known as the zerobias anomaly (ZBA) (see Fig. 1). In the case of multiple mutually interacting impurities, the DOS is more complex and depends on a competition between $T_{\mathrm{K}}$ and a pairwise inter-impurity exchange interaction $J_{12}$ (refs 10,11). For $\left|J_{12}\right| \gg k_{\mathrm{B}} T$, where $T$ is the temperature, the Kondo resonance is suppressed at energy scales $\lesssim\left|J_{12}\right|$ (Fig. 1), resulting in a split ZBA at zero external magnetic field, which acts as an indicator of spontaneous spin polarization and static magnetic phases within the system ${ }^{12-14}$.
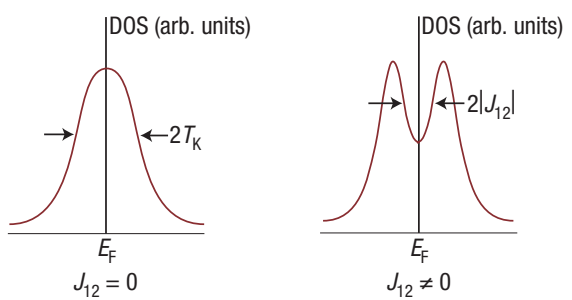

Figure 1 Kondo resonance in the density of states (DOS) of a multiple-spin system with zero and non-zero inter-impurity exchange interaction $J_{12}$. $J_{12}$ is negative for ferromagnetic and positive for antiferromagnetic exchange. In either case, magnetic order sets in when $\left|J_{12}\right| \gg k_{\mathrm{B}} T$, which suppresses the single-impurity Kondo resonance at the energy scale (defined by the Kondo temperature, $\left.T_{\mathrm{K}}\right) \lesssim\left|J_{12}\right|$, leading to a gap at the Fermi energy, $E_{\mathrm{F}}$.

Along with the magnetic effects, the embedded localized states also act as scattering centres for conduction electrons, leading to back-scattering and interference effects when the phase coherence length of the electrons exceeds the inter-impurity distance $(R)$. A completely random spatial distribution of the impurities gives rise to weak localization phenomena at low $T$, which are manifest as a universal aperiodic fluctuation in the linear magnetoconductance as a function of either $E_{\mathrm{F}}$ or a weak transverse magnetic field $\left(B_{\perp}\right)$ (ref. 15). However, when the impurities are arranged in a quasi-regular manner, the transport takes place through multiple, connected Aharonov-Bohm rings encircling a discrete number of impurities. As a function of $B_{\perp}$, the magnetoconductance at low $T$ breaks into quasi-periodic phase-coherent oscillations with periodicity given by

$$
\Delta B_{\perp} \approx h / \pi e R_{\mathrm{C}}^{2}
$$

where $R_{\mathrm{C}}$ is the radius of a stable ring ${ }^{16,17}$.

Observation of Kondo resonance in unconfined mesoscopic one-dimensional $^{7}$ or two-dimensional ${ }^{8,9}$ devices from highmobility semiconductor heterostructures indicates unexpected localized spin states, whose microscopic origin is not understood. Most experimental evidence so far is extracted from the ZBA in non-equilibrium transport, which remains inconclusive on the spatial layout, or the mutual interaction of the states. Here, we 

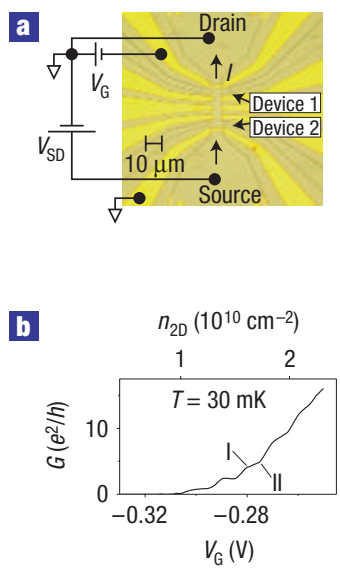

$c$
兘
क

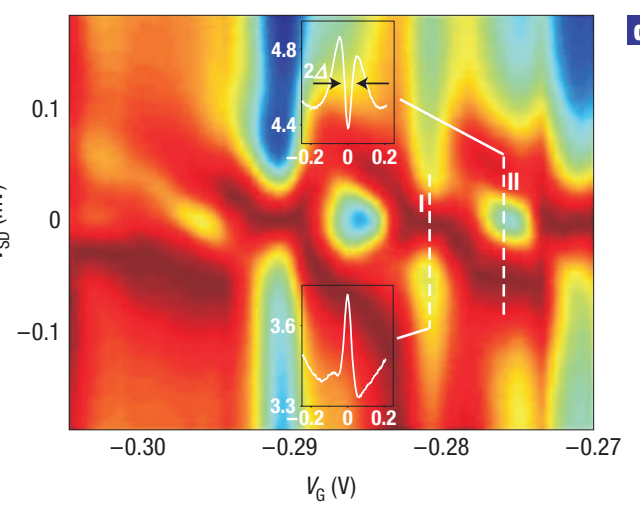

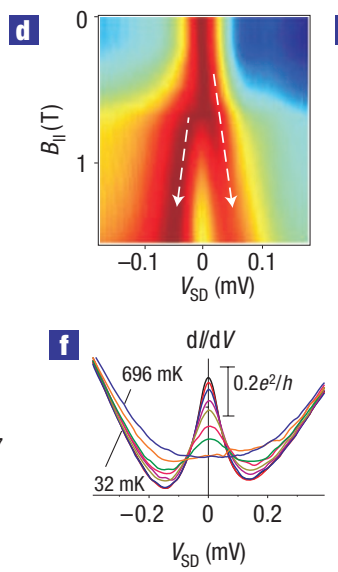

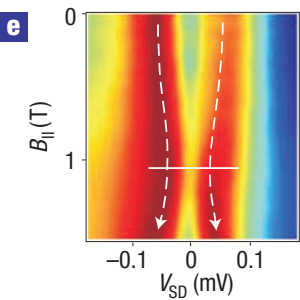

g

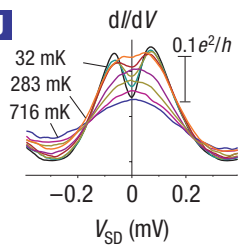

Figure 2 Non-equilibrium characteristics. a, A set of two typical devices and electrical connections. The active area of a device is defined by the gate-covered region of the etched mesa. The data shown here were obtained from device 1 , with device 2 and other side gates kept grounded. $\mathbf{b}$, Typical linear conductance, $G$, versus gate voltage $V_{G}$ (and electron density $n_{2 \mathrm{D}}$ ) for device 1 at $30 \mathrm{mK}$ and zero external magnetic field. c, Surface plot of differential conductance $\mathrm{d} / / \mathrm{d} V$ of device 1 in the $V_{S D}-V_{G}$ plane. Each $\mathrm{d} / / \mathrm{d} V-V_{\mathrm{SD}}$ trace at a particular $V_{\mathrm{G}}$ was vertically shifted for levelling. The insets illustrate ZBA-I- and ZBA-II-type resonances at points I and II in Fig. $1 \mathrm{~b}$, respectively. d, Surface plot of ZBA-I in in-plane magnetic field $\left(B_{\|}\right)$. The dashed arrows show a linear monotonic splitting with effective $g$-factor $\left|g^{*}\right| \approx 0.5$, which confirms the role of spin. e, Surface plot of ZBA- $\|$ in $B_{\|}$. The single-impurity Kondo behaviour dominates above $B_{\|} \sim \Delta / g^{*} \mu_{\mathrm{B}}$ (the horizontal line), where $\Delta$ is the half-gap defined in the text and $\mu_{\mathrm{B}}$ is the Bohr magneton. f, Monotonic temperature $(T)$ suppression of ZBA-I. $\mathbf{g}, T$ dependence of $\mathrm{d} l / \mathrm{d} V$ at ZBA-Il. $\mathrm{d} l / \mathrm{d} V$ is non-monotonic in $T$ for $\left|V_{\mathrm{SD}}\right| \lesssim \Delta$.

have exploited both spin and orbital effects of localized states by combining the investigation of non-equilibrium characteristics and linear magnetotransport in the same mesoscopic device. The results are not only consistent with the existence of local magnetic moments in high-mobility heterostructures, but also, for the first time, indicate the nature of mutual interaction, and a plausible microscopic origin of these moments.

Our mesoscopic devices were fabricated from Si-modulationdoped GaAs/AlGaAs heterostructures, where unintentional magnetic impurities are expected to be absent, and a high low- $T$ mobility of $\sim 1-3 \times 10^{6} \mathrm{~cm}^{2} \mathrm{~V}^{-1} \mathrm{~s}^{-1}$ provides a long as-grown elastic mean free path $\sim 6-8 \mu \mathrm{m}$. The lithographic dimensions of our devices were kept smaller than this length scale to ensure quasi-ballistic transport (Fig. 2a). Non-equilibrium transport in these systems involves measuring the differential conductance $\mathrm{d} I / \mathrm{d} V$ as a function of $V_{\mathrm{SD}}$ at fixed voltages $\left(V_{\mathrm{G}}\right)$ applied on the (non-magnetic) surface gate, which reflects the low-energy DOS through the Landauer formalism. Experiments were carried out at electron temperatures as low as $\approx 30 \mathrm{mK}$.

Below $\sim 100 \mathrm{mK}$, the zero-field $\mathrm{d} I / \mathrm{d} V$ shows rich structures in the $V_{\mathrm{G}}-V_{\mathrm{SD}}$ plane (Fig. 2c). For most devices, the structures are strongest in the electron-density range $n_{2 \mathrm{D}} \sim 1-3 \times 10^{10} \mathrm{~cm}^{-2}$ (Fig. 2b), and often visible up to $G \gtrsim 10-15 \times\left(e^{2} / h\right)$, where $G=\mathrm{d} I / \mathrm{d} V$ at $V_{\mathrm{SD}}=0$. In Fig. $2 \mathrm{c}, \mathrm{d} I / \mathrm{d} V$ consists of a repetitive sequence of two types of resonance as $V_{\mathrm{G}}$ is increased. We denote the single-peak resonance as ZBA-I, which splits intermittently to form a gap at $E_{\mathrm{F}}$, referred to as ZBA-II. We define $\Delta$ as the half-width at half-depth of ZBA-II. Similar non-equilibrium characteristics were observed in over 50 mesoscopic devices from five different wafers, where reducing the setback distance $\left(d_{\mathrm{s}}\right)$ below $\sim 60-80 \mathrm{~nm}$ was generally found to have a detrimental effect on the clarity of the resonance structures, often leading to broadening or complete suppression of both types of ZBA.

The ZBAs are logarithmically suppressed for $T \gtrsim 300 \mathrm{mK}$ (Fig. 2f,g), and split linearly in an in-plane magnetic field when Zeeman energy exceeds the corresponding $\Delta$ (Fig. 2d,e). These are characteristic features of Kondo resonance ${ }^{9}$. (See Supplementary
Information for arguments against alternative explanation of the ZBA.) Moreover, both linear and nonlinear transport at ZBA-II indicate non-monotonicity at the scale of $\Delta$ (Fig. 2e,g), which can be understood in a 'two-impurity Kondo' model that implies the presence of multiple interacting spins ${ }^{9,13}$. Because $\Delta \sim\left|J_{12}\right|$ (Fig. 1), the re-entrant splitting of ZBA in Fig. $2 \mathrm{c}$ thus indicates $J_{12}$ to be oscillatory in $V_{\mathrm{G}}$, where ZBA-I corresponds to $\left|J_{12}\right| \ll k_{\mathrm{B}} T$.

Before exploring the microscopic origin of $J_{12}$, we discuss the linear low-transverse-field magnetoconductance measurements, which provide information on the spatial layout of the scattering centres. At the lowest $T$, mesoscopic devices that show clear resonances in $\mathrm{d} I / \mathrm{d} V$ also show reproducible quasi-periodic oscillations in the linear magnetoconductance over a transverse field range $\left|B_{\perp}\right| \lesssim 0.1 \mathrm{~T}$. These oscillations appear over the same range of $n_{2 \mathrm{D}}$ as the ZBA, but, apart from being slightly smaller in amplitude near a ZBA-II, they are mostly insensitive to the details of the ZBA structure. In Fig. 3a, b this is illustrated with a device of configuration similar to that shown in Fig. 2a. Discrete peaks in the fast-Fourier power spectrum of these oscillations imply their quasi-periodicity, thereby distinguishing them from universal conductance fluctuations.

To analyse the magnetoconductance oscillations, we note that the strong peak at $1 / \Delta B_{\perp} \approx 105 \mathrm{~T}^{-1}$ (Fig. 3c) is common to all traces and corresponds to the 'unit cell' in the scatterer array, which is an Aharonov-Bohm ring encircling a single scattering centre, with a radius of $R / 2$ (orbit (ii)). From equation (1), we get $R \approx 670 \pm 30 \mathrm{~nm}$, which was found to be weakly device dependent, varying between 600 and $800 \mathrm{~nm}$, but insensitive to the lithographic dimensions of the devices. Often peaks at higher frequency would also appear, consistent with Aharonov-Bohm rings encircling larger numbers of scattering centres (see orbit (iii) in Fig. 3c). The peak at lower frequency, which becomes stronger at $\left|B_{\perp}\right| \gtrsim 0.08 \mathrm{~T}$ (fast-Fourier power spectrum denoted by empty blue circles in Fig. 3c), represents orbit (i), which is inscribed by the finite cross-section of the scattering centres.

Implicitly, the phase-coherent oscillations indicate a quasiregular distribution of the scattering centres. We independently cross-checked this from commensurability of classical electron 

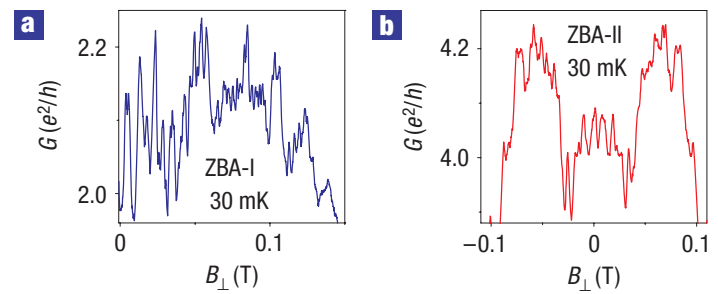

G

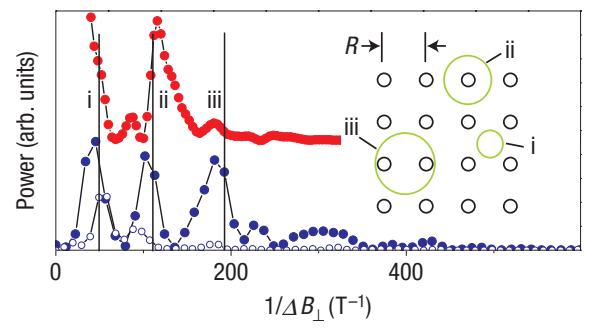

d

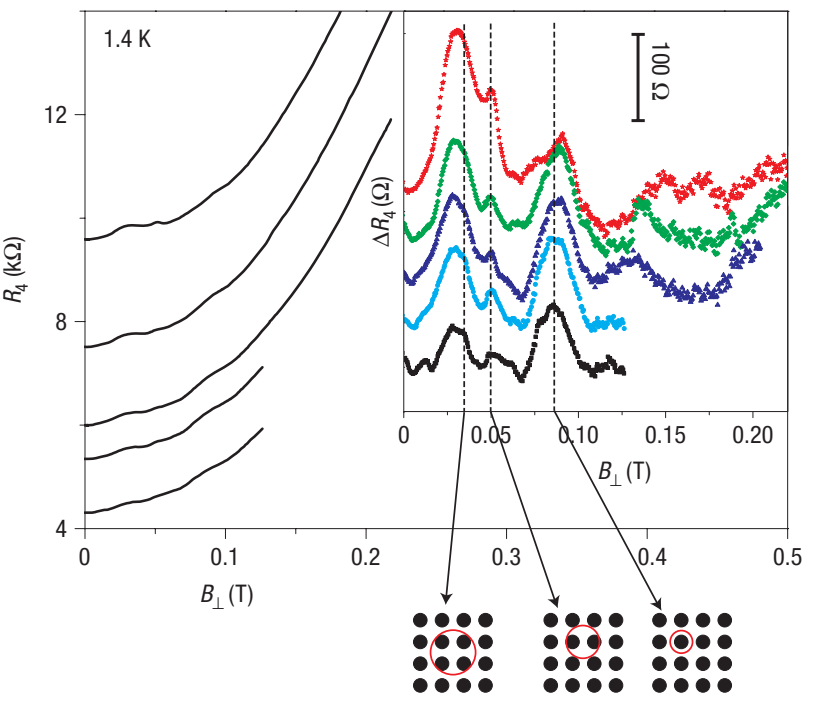

Figure 3 Quantum and classical magnetotransport in perpendicular magnetic field $\left(B_{\perp}\right)$. a,b, Typical linear magnetoconductance $G$ oscillation at a single-peak resonance (ZBA-I) (a) and a double-peak resonance (ZBA-II) (b). c, Power spectra of the magnetoconductance oscillations. The filled symbols (blue, ZBA-l; red, ZBA-II) represent spectra obtained from the range $\left|B_{\perp}\right| \leq 0.065 \mathrm{~T}$, whereas the open (blue) symbols represent the spectrum (vertically scaled for clarity) from

$0.065 \mathrm{~T}<B_{\perp}<0.15 \mathrm{~T}$. The orbits corresponding to the peaks are indicated in the inset schematic diagram. $\mathbf{d}$, Four-probe linear magnetoresistance $R_{4}$ at $1.4 \mathrm{~K}$ for five electron densities from 1.22 (topmost trace) to $1.39 \times 10^{10} \mathrm{~cm}^{-2}$ (bottom trace), where well-defined ZBAs appear at low temperatures. Inset: Magnetoresistance after subtracting the parabolic background. The dashed lines, which denote various commensurate orbits, are computed using the average density of $1.31 \times 10^{10} \mathrm{~cm}^{-2}$, and radius $R \approx 500 \mathrm{~nm}$.

trajectories in a two-dimensional array of point scatterers at finite $B_{\perp}$ (ref. 18). In Fig. 3d, we show the four-probe magnetoresistance of the same device at $T \simeq 1.4 \mathrm{~K}$, which consists of small peaklike structures superposed on a parabolically increasing background. On subtracting the background, the signature of commensurable orbits at $B_{\perp} \approx 0.08,0.05$ and $0.03 \mathrm{~T}$ can be observed, corresponding to the cyclotron radius of the electron encircling one, two and four scattering centres respectively (inset). This gives $R \sim 500 \mathrm{~nm}$, consistent with the estimate obtained from phase-coherent oscillations.

Assuming each scattering centre hosts a localized spin state, we find that $J_{12}$ can be naturally associated with RKKY-type indirect exchange, which is expected in a system composed of itinerant electrons and localized magnetic impurities. In this framework, the oscillatory behaviour of $J_{12}$ in $V_{\mathrm{G}}$ arises from the range function $\Psi\left(2 k_{\mathrm{F}} R\right)$ in the interaction magnitude, which reverses its sign with a periodicity of $\pi$ in $2 k_{\mathrm{F}} R, k_{\mathrm{F}}=\sqrt{2 \pi n_{2 \mathrm{D}}}$ being the Fermi wavevector and $R$ being fixed for a given device. Analytically,

$$
\Delta \sim\left|J_{12}\right| \sim E_{\mathrm{F}}(\Gamma / U)^{2}\left|\Psi\left(2 k_{\mathrm{F}} R\right)\right| .
$$

Figure $4 \mathrm{a}$ shows the direct confirmation of this, where we have plotted $\Delta$ as a function of $2 k_{\mathrm{F}} R$ for the device in Fig. $2 \mathrm{c}$. The clear periodicity of $\approx \pi$ (within $\pm 5 \%$ ) in $2 k_{\mathrm{F}} R$ can be immediately recognized as the so-called ' $2 k_{\mathrm{F}} R$ oscillations' in the RKKY interaction, establishing the multiple-localized-spin picture.

The absolute magnitude of $J_{12}$, and hence $\Delta$, for a twodimensional distribution of spins may differ widely from the straightforward pairwise RKKY interaction, and would be affected by frustrated magnetic ordering or spin-glass freezing ${ }^{19}$, as well as deviation from perfect periodicity in the spin arrangements ${ }^{20}$. Nevertheless, a framework for comparison of $\Delta$ in different samples can be obtained from equation (2) by normalizing $\Delta$ with $E_{\mathrm{F}}$. As shown in Fig. 4b, manually adjusting for the experimental
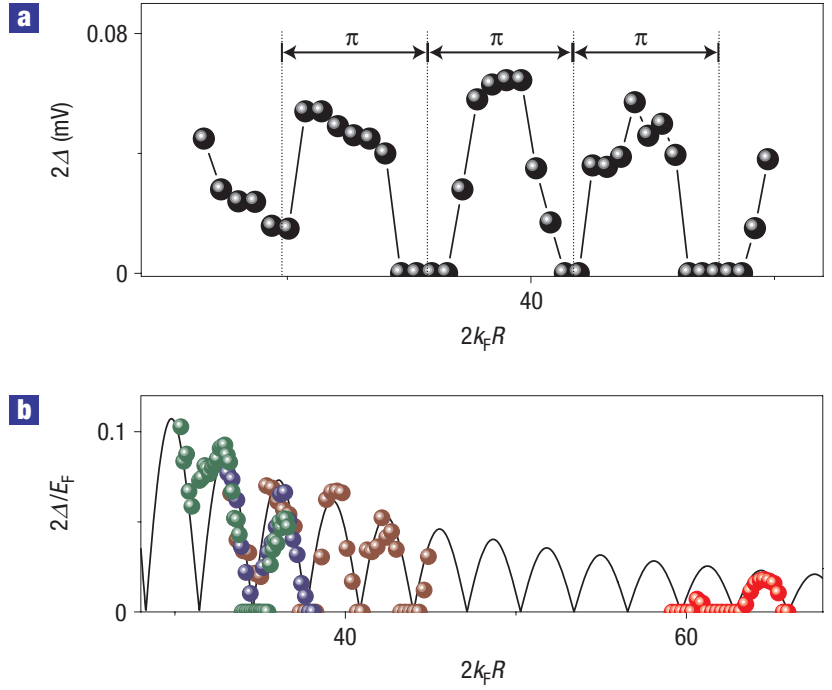

Figure 4 RKKY indirect exchange and ' $2 k_{\mathrm{F}} \boldsymbol{R}$ oscillations'. a, $2 \Delta$ from Fig. $2 \mathrm{c}$ as a function of $2 k_{\mathrm{F}} R$, where $k_{\mathrm{F}}$ is the Fermi wavevector, and $R$ is the inter-spin distance obtained from magnetoconductance oscillations. $\mathbf{b}, 2 \Delta / E_{\mathrm{F}}$ as a function of $2 k_{\mathrm{F}} R$ for four different devices. The solid line is proportional to the pairwise RKKY range function (see the text). Local disorder governs the experimentally attainable range of $2 k_{\mathrm{F}} R$ within a given mesoscopic device.

uncertainty in $k_{\mathrm{F}}$ and $R, \Delta / E_{\mathrm{F}}$ for four different devices with various lithographic dimensions can be made to collapse onto the solid line proportional to the modulus of the two-impurity RKKY range function over a wide range of $2 k_{\mathrm{F}} R$ (ref. 21). 
A plausible mechanism for local moment formation in high-mobility GaAs/AlGaAs systems would thus require us to simultaneously account for (1) the observed magnitude of $R$ and the quasi-regular impurity distribution and (2) Kondo coupling of the localized spin to the conduction electrons. We show below that background potential fluctuations arising from a strongly correlated dopant layer can address both observations quantitatively. In this scheme, which is similar to moment formation at metal-semiconductor Schottky barriers ${ }^{6}$, depletion of electrons at strong potential fluctuations leads to Mott transition locally around the depletion region. Isolated localized states are formed, which become magnetic when $\Gamma / U$ is reduced below $\sim 0.1$ (the Mott criterion) with decreasing $n_{2 \mathrm{D}}$.

For heavily compensated donor layers of monolayer-doped heterostructures, the density correlation function $\phi(q)$ is commonly approximated as that of a non-ideal plasma ${ }^{22,23}$ :

$$
\phi(q)=\frac{N_{\delta} q}{q+\left(2 \pi N_{\delta} e^{2} / \epsilon_{0} \epsilon_{\mathrm{r}} k_{\mathrm{B}} T_{0}\right)\left[1-\exp \left(-2 q d_{\mathrm{s}}\right)\right]},
$$

which in the long-wavelength limit, $q \ll 1 / d_{\text {s }}$, leads to an effective dopant concentration of

$$
N_{\text {eff }}=\epsilon_{0} \epsilon_{\mathrm{r}} k_{\mathrm{B}} T_{0} / 4 \pi e^{2} d_{\mathrm{s}},
$$

where $\epsilon_{0}$ is the vacuum permittivity, $\epsilon_{\mathrm{r}}$ is the relative permittivity and $N_{\delta}$ and $T_{0}$ are the bare dopant density and freezing-out temperature for electrons left in the dopant layer, respectively. Strong Coulomb interaction can further lead to an ordering in this effective donor distribution, with a sharp peak in the correlation function at $q=q_{\max } \approx \sqrt{N_{\text {eff }}}$ (ref. 24). Using known system parameters $T_{0} \approx 80 \mathrm{~K}$ and $d_{\mathrm{s}}=80 \mathrm{~nm}$, we find that strong background potential fluctuations are expected at a length scale of $1 / q_{\max } \sim 0.4-0.5 \mu \mathrm{m}$, which agrees well with the observed magnitude of $R$, as well as results of direct experimental imaging ${ }^{25}$. We note that both phenomena will be observable as long as any deviation $(\delta R)$ from a perfect ordering satisfies $\delta R \ll 2 \pi / k_{\mathrm{F}}$ for RKKY dynamics, or $\delta\left(R^{2}\right) \ll(h / e) / \Delta B_{\perp}$ for the phasecoherent oscillations. These conditions impose $\delta R / R \ll 0.4$, which is realistic, because following ref. 24 we obtain $\delta R / R \sim \delta q / q_{\max } \lesssim 0.2$.

To verify the Kondo coupling of localized moments to surrounding itinerant electrons, we have calculated the ratio $\epsilon / \Gamma$ for the localized states using the experimentally observed half-width of the ZBA-I resonances, and equating it to $T_{\mathrm{K}}$. From the trace shown in the lower inset of Fig. $2 \mathrm{c}$, $T_{\mathrm{K}} \approx 0.035 \mathrm{mV} \sim E_{\mathrm{F}} \exp (\pi \epsilon / 2 \Gamma)$ in the $U \rightarrow \infty$ limit, which gives $\epsilon / \Gamma \approx-2.1$, confirming the Kondo regime. (Here $U \sim e^{2} / \xi \gg E_{\mathrm{F}}$, and $\xi \sim 150 \mathrm{~nm}$ is the localization length obtained experimentally from the magnetoconductance oscillations corresponding to orbit (i) in Fig. 3c.) Notably, formation of magnetic quantum point contacts c $^{7,26}$ in a percolation-type representation of the disorder landscape at low $n_{2 \mathrm{D}}$ (ref. 28) cannot explain the commensurability effect or phase-coherent magnetoconductance oscillations shown in Fig. 3, as they require extended and uninterrupted electron orbits.

\section{METHODS}

\section{WAFER AND DEVICE CHARACTERISTICS}

Devices were fabricated from (100) molecular beam epitaxy-grown GaAs $/ \mathrm{Al}_{0.33} \mathrm{Ga}_{0.66}$ As heterostructures, where the two-dimensional electron layer was formed $300 \mathrm{~nm}$ below the surface. A setback distance of $d_{\mathrm{s}}=80 \mathrm{~nm}$ was obtained by inserting an undoped layer of $\mathrm{Al}_{0.33} \mathrm{Ga}_{0.66}$ As between the $\mathrm{Si}$ $\delta$-doped layer, with dopant density $N_{\delta} \approx 2.5 \times 10^{12} \mathrm{~cm}^{-2}$, and the heterointerface. This resulted in a heavily compensated dopant layer with a filling factor $f \approx 0.9$. The device width was determined by the width of the wet-etched mesa, and fixed at $\approx 8 \mu \mathrm{m}$, and the length defined by the width of the surface gate was varied between 2 and $7 \mu \mathrm{m}$ (Fig. 2a).

\section{MEASUREMENTS}

Non-equilibrium measurements were carried out inside a dilution refrigerator (base electron temperature $\approx 30 \mathrm{mK}$ ), with an ac $+\mathrm{dc}$ two-probe technique. The ac modulation $(\sim 2-5 \mu \mathrm{V}$ at $90 \mathrm{~Hz})$ was kept much less than $k_{\mathrm{B}} T$ to avoid heating or other nonlinearities. Before measurement all devices were subjected to slow cooling cycles between room temperature and $4.2 \mathrm{~K}$, with each cooling lasting $5-10 \mathrm{~h}$. See Supplementary Information for more details on the effect of cooling and disorder.

\section{Received 9 August 2006; accepted 11 January 2007; published 11 March 2007.}

\section{References}

1. Goldhaber-Gordon, D. et al. Kondo effect in a single-electron transistor. Nature 391, 156-159 (1998)

2. Cronenwett, S. M., Oosterkamp, T. H. \& Kouwenhoven, L. P. A tunable Kondo effect in quantum dots. Science 281, 540-544 (1998).

3. Jeong, H., Chang, A. M. \& Melloch, M. R. The Kondo effect in an artificial quantum dot molecule. Science 293, 2221-2223 (2001)

4. Craig, N. J. et al. Tunable nonlocal spin control in a coupled-quantum dot system. Science $\mathbf{3 0 4}$ 565-567 (2004)

5. Schrieffer, J. R. \& Wolff, P. A. Relation between the Anderson and Kondo hamiltonians. Phys. Rev. 149, 491-492 (1966)

6. Wolf, E. L. \& Losee, D. L. Spectroscopy of Kondo and spin-flip scattering: High-field tunneling studies of Schottky-Barrier junctions. Phys. Rev. B 2, 3660-3687 (1970).

7. Cronenwett, S. M. et al. Low-temperature fate of the 0.7 structure in a point contact: A Kondo-like correlated state in an open system. Phys. Rev. Lett. 88, 226805 (2002).

8. Ghosh, A., Ford, C. J. B., Pepper, M., Beere, H. E. \& Ritchie, D. A. Possible evidence of a spontaneous spin polarization in mesoscopic two-dimensional electron systems. Phys. Rev. Lett. 92, 116601 (2004).

9. Ghosh, A. et al. Zero-bias anomaly and kondo-assisted quasiballistic 2D transport. Phys. Rev. Lett. 95, 066603 (2005).

10. Jayaprakash, C., Krishnamurthy, H. R. \& Wilkins, J. W. Two-impurity Kondo problem. Phys. Rev Lett. 47, 737-740 (1981)

11. Affleck, I. \& Ludwig, A. W. W. Exact critical theory of the two-impurity Kondo model. Phys. Rev. Lett. 68, 1046-1049 (1992).

12. Pasupathy, A. N. et al. The Kondo effect in the presence of ferromagnetism. Science 306, 86-89 (2004)

13. Heersche, H. B. et al. Kondo effect in the presence of magnetic impurities. Phys. Rev. Lett. 96, 017205 (2006).

14. Nygard, J., Cobden, D. H. \& Lindelof, P. E. Kondo physics in carbon nanotubes. Nature 408 $342-346(2000)$

15. Thornton, T. J., Pepper, M., Ahmed, H., Davies, G. J. \& Andrews, D. Universal conductance fluctuations and electron coherence lengths in a narrow two-dimensional electron gas. Phys. Rev. B 36, 4514-4517 (1987).

16. Schuster, R., Ensslin, K., Wharam, D., Kühn, S. \& Kotthaus, J. P. Phase-coherent electrons in a finite antidot lattice. Phys. Rev. B 49, 8510-8513 (1994).

17. Weiss, D. et al. Quantized periodic orbits in large antidot arrays. Phys. Rev. Lett. 70, 4118-4121 (1993).

18. Weiss, D. et al. Electron pinball and commensurate orbits in a periodic array of scatterers. Phys. Rev. Lett. 66, 2790-2793 (1991)

19. Hirsch, M. J., Holcomb, D. F., Bhatt, R. N. \& Paalanen, M. A. ESR studies of compensated Si:P,B near the metal-insulator transition. Phys. Rev. Lett. 68, 1418-1421 (1992).

20. Roche, S. \& Mayou, D. Formalism for the computation of the RKKY interaction in aperiodic systems. Phys. Rev. B 60, 322-328 (1999).

21. Béal-Monod, M. T. Ruderman-Kittel-Kasuya-Yosida indirect interaction in two dimensions. Phys. Rev. B 36, 8835-8836 (1987)

22. Efros, A. L., Pikus, F. G. \& Samsonidze, G. G. Maximum low-temperature mobility of two-dimensional electrons in heterojunctions with thick spacer layer. Phys. Rev. B 41, 8295-8301 (1990).

23. Buks, E., Heiblum, M. \& Shtrikman, H. Correlated charged donors and strong mobility enhancement in a two-dimensional electron gas. Phys. Rev. B 49, 14790-14793 (1994).

24. Grill, R. \& Döhler, G. H. Effect of charged donor correlation and Wigner liquid formation on the transport properties of a two-dimensional electron gas in modulation $\delta$-doped heterojunctions. Phys. Rev. B 59, 10769-10777 (1999).

25. Finkelstein, G., Glicofridis, P. I., Ashoori, R. C. \& Shayegan, M. Topographic mapping of the quantum Hall liquid using a few-electron bubble. Science 289, 90-94 (2000).

26. Graham, A. C., Pepper, M., Simmons, M. Y. \& Ritchie, D. A. Anomalous spin-dependent behavior of one-dimensional subbands. Phys. Rev. B 72, 193305 (2005).

27. Berggren, K.-F., Jaksch, P. \& Yakimenko, I. Effects of electron interactions at crossings of Zeeman-split subbands in quantum wires. Phys. Rev. B 71, 115303 (2005).

28. Meir, Y. Percolation-type description of the metal-insulator transition in two dimensions. Phys. Rev. Lett. 83, 3506-3509 (1999).

Acknowledgements

The work was supported by an EPSRC funded project. A.G. acknowledges discussions with

C. J. B. Ford, G. Gumbs, M. Stopa, P. B. Littlewood, H. R. Krishnamurthy, B. D. Simons, C. M. Marcus, D. Goldhaber-Gordon and K. F. Berggren. C.S. acknowledges financial support from the Gottlieb Daimler and Karl Benz Foundation.

Correspondence and requests for materials should be addressed to C.S. or A.G.

Supplementary Information accompanies this paper on www.nature.com/naturephysics.

Author contributions

Experimental work and data analysis were carried out by C.S. and A.G., project planning by A.G. and M.P. and wafer growing by I.F. and D.A.R.

Competing financial interests

The authors declare no competing financial interests.

Reprints and permission information is available online at http://npg.nature.com/reprintsandpermissions/ 